\title{
A Platform for Exploring Reconfigurability in Distributed and Disaggregated Wireless Networks
}

\author{
Linda Doyle \\ Centre for Telecommunications Value-Chain Research \\ Trinity College, University of Dublin, Ireland \\ Email: ledoyle@ctvr.ie
}

\begin{abstract}
The purpose of this paper is to present an overview of a platform for exploring reconfigurability in wireless networks. The types of networks that are particularly of interest here are distributed and disaggregated networks. The term disaggregated is used to describe networks that are not alone distributed but also non-homogenous and controlled by different and possibly competing entities. In distributed and disaggregated networks, network-wide reconfiguration, if indeed possible, will not be achieved through a co-ordinating or controlling entity as a global view or global control of the network does not exist. Therefore mechanisms for facilitating network-wide reconfiguration based on local knowledge only are needed. The platform presented in the paper combines research outcomes from ad hoc networking research and work carried out in the area of reconfigurable radio design in an effort to address these issues.
\end{abstract}

\section{INTRODUCTION}

This paper focuses on reconfiguration in distributed and disaggregated wireless networks. The term disaggregated is used to describe networks that are not alone distributed but also non-homogenous and controlled by different and possibly competing entities. The term reconfigurable is used in a very general sense. It includes reconfiguration for self-organization purposes, as for example in the case of a group of first time nodes auto-configuring. It includes optimization based reconfiguration, as for example in the case of a network optimizing its performance through making better use of radio resources. It includes reconfigurability for the purposes of updating standards or reconfigurability as a means of providing wider user choice etc. Reconfigurability is not just confined to parameters of the physical layer but to all higher layers of the system as well.

As a means of prefacing this work it is useful to consider a conceptual representation of the reconfiguration process of a node in a distributed or disaggregated wireless system. In Figure 1 a minimalist state diagram is shown. The node is, on one level, an independent entity and can make choices in its own interest but on another level, the node is part of a community and must not cause conflict. The reconfiguration process can be considered to have a minimum of four states. (1) The first state is the normal operating state during which the node is operating as configured. It is assumed in our discussions that a node is capable of making observations. The term contextual observations is used to emphasise that the state the node is operating in, will have a bearing on what observations can be made. (2) On the receipt of what

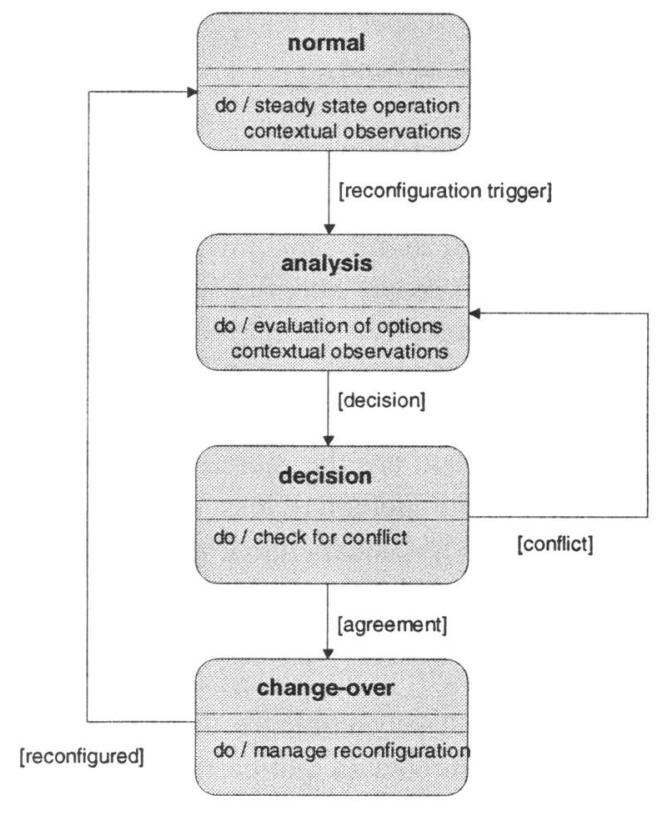

Fig. 1. The Reconfiguration Process

we call a 'reconfiguration trigger' the node enters an analysis state to determine how it should best react. During this state the node analyses possible courses of action, i.e. possible reconfiguration options. (3) Once analysis is complete the node makes a decision as to how to react, i.e. chooses a preferred reconfiguration option (or of course chooses to stay as is). The decision state may be a stable state or the node may have to revisit its decision on the basis that its decision conflicts with the decision of others. (4) Once a stable decision is reached the node moves to the changeover state during which the reconfiguration process takes place. The key here is to reconfigure and reach stability as efficiently as possible. Once completed, the node enters the state of normal operation again.

With this definition of reconfigurability, everything in the network now becomes a variable that can set to an optimal value to suit current conditions (e.g. network conditions, channel conditions, business conditions etc.) In such a highly reconfigurable network, the value of the variable (e.g. modulation scheme, frequency of operation, routing protocol, security level etc.) can be determined by each node in the network. Obviously some decisions are unilateral and a node can simply set the variable to the value desired without any need for 
consultation with others. Others are multilateral and consensus must be reached before any changes to the new value of the variable are made. In distributed and disaggregated networks reaching global consensus when many nodes only have local views is a challenge.The purpose of this paper is to introduce a highly flexible platform that can facilitate the exploration of reconfigurability, allowing nodes to function as described in Figure 1 , at all layers of a communication system at both the node and the network level.

The remainder of the paper is organized as follows: Section II introduces the exploration platform, a platform which draws on work from the fields of ad hoc networking and software radio. Following the description of the platform two examples of how the platform is used are briefly presented. In Section III a unilateral reconfiguration scenario is presented and in Section IV a multilateral example is given. The final section of the paper, Section V, concludes.

\section{ThE EXPLORATION PlatForm}

A key to investigating many of the interesting questions in the area of wireless reconfigurable networks is to provide a suitable research platform that is flexible enough to facilitate a wide range of reconfigurability at multiple levels and granularities. The reconfiguration platform and focus of this paper, is a combination of an ad hoc network research platform known as DAWN (Dublin Ad hoc Wireless Network) and a software radio engine known as IRIS (Implementing Radio in Software) with enhanced features.

\section{A. DAWN}

DAWN was originally created for the purposes of facilitating research in the area of ad hoc networking [1]. Details of DAWN can be found in [2]. At the core of DAWN is a dynamic modular communication stack that runs on each of the nodes of the ad hoc network. Layers of the stack can be independently designed in a standalone fashion. A generic layer interface allows the dynamic assembly of these layers to form a network communication stack consisting of the relevant hardware and software elements. The interlayer interface is very simple, consisting of primitives to send information upwards or downwards through the stack. A wide range of layers have been designed for DAWN. A Windows CE version of everything exists for handheld devices.

\section{B. IRIS}

The reconfigurable radio consisting of a general-purpose processor software radio engine, known as IRIS (Implementing Radio in Software) [3] and a minimal hardware frontend was originally created for facilitating research in the area of software radio. The fundamental unit for building reconfigurable radios in the IRIS Radio Architecture is the Radio Component (a unit of radio functionality). A Radio Component is the basic unit of an IRIS mplementation and comprises an individual stage in the signal-processing chain of an IRIS reconfigurable radio. The actual level of functional complexity that a Radio Component may encapsulate is at the discretion of the Radio Component designer. A radio consists of a string of Radio Components. Users either make use of existing Radio Components that have already been created as part of the research process or users design new components. An XML file is used to describe how the Radio Components connect together to form the radio of interest. Initial Radio Component parameters can also be set using the XML file.

IRIS parses the XML file and the IRIS Component Manager loads the Radio Components specified in the XML configuration file and unloads a previously loaded radio configuration. The Component Manager also compiles an inventory of Components which may be located either on the host PC or in a remote location connected via Ethernet or internet, and these comprise the available Components that may be used as part of a radio implementation. The IRIS Radio Engine implements the radio.

The key feature of IRIS is that it supports real-time reconfiguration of the radio. IRIS uses a Control Logic Manager (CLM) which is the main means of reconfiguring a radio configuration when the radio is in operation. The CLM is independent of the Components (i.e. the CLM is a separate process that connects to the radio using a common interface) and therefore may externally modify the paramters, structure and operation of any of the Components that comprise the radio implementation. IRIS supports three levels of reconfiguration [3]. The first, parametric reconfiguration, involves the dynamic alteration of individual parameters of signal processing functionality (e.g.change of filter cutoff points). The second, structural reconfiguration, involves the alteration of the layout of the radio system or the replacement of some aspect of the software of the system while still performing the same overall application (e.g. change of modulation scheme). The third, application reconfiguration involves completely replacing the software of the software radio with an entirely different software radio configuration (e.g. change from GSM to WLAN). The hardware associated with IRIS consists of a minimal RF front-end chosen as appropriate, ADC/DAC hardware and a WaveRunner Plus 253 Peripheral Component Interconnect (PCI) transceiver board manufactured by Red River.

\section{The Melding of DAWN and IRIS}

The reconfiguration platform and focus of this paper, is a combination of the work from DAWN and IRIS with enhanced features. The diagram in Figure 2 shows the structure of a typical node in the reconfigurable exploration platform. At the centre is the DAWN stack. IRIS takes the role of a new physical layer, adding an extra dimension to the flexibility of DAWN. The IRIS API [3] allows for this type of integration. The platform also features an item referred to as a blackboard. The blackboard is used to facilitate reconfiguration of the node through the use of a cross-layer optimiszation/reconfiguration approach. Cross-layer reconfiguration typically involves designing application-driven, adaptive and resource-aware layers that can benefit from sharing information across the protocol stack. In our platform the blackboard provides a means of 


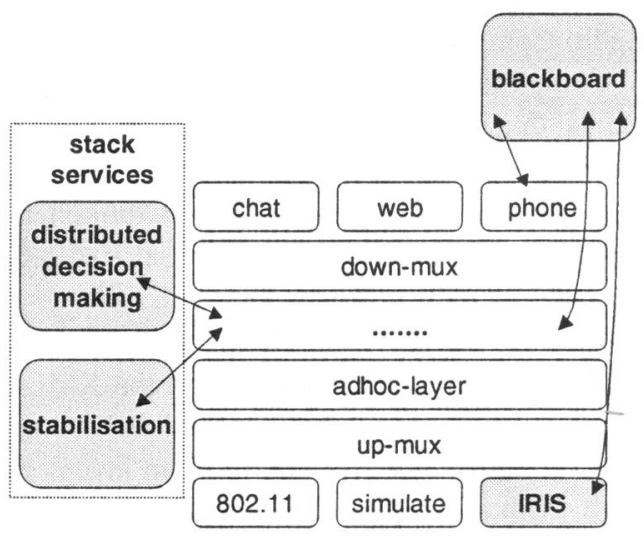

Fig. 2. The Reconfiguration Exploration Platform

passing information between layers. Channel state information for example can be placed on the blackboard and the application can take account of this if it so chooses. Information can also flow in the opposite direction, so for example the radio can adapt to the needs of the application. Using this mechanism different parameters in the layers of the stack can be reconfigured to achieve a desired node objective. A mechanism also exists, though not highlighted in the diagram, for exchanging layers of the stack in real time so not alone can parameters within layers be reconfigured, whole layers can be changed.

On the left of Figure 2 are a group of features that fall under the heading stack services. These are called upon when multilateral decisions must be made. In these cases there are likely to be divergent systems views and conflict must either be avoided or resolved. There are two stack services to address this. The first stack service provides a means of making the decision. Distributed consensus protocols, typically provide solutions for reaching agreement among remote processes (e.g. nodes of a newtork). However in highly distributed and/or disaggregated wireless and mobile networks consensus algorithms must overcome difficulties introduced by asynchrony (e.g. asynchronous message passing), limited local knowledge, variable link quality, unstable links and sometimes also deal with the restrictions of limited bandwidth and as a result their usefulness is limited [4]. Therefore rather than use distributed consensus protocols we have developed two different mechanisms (a Diffusion of Innovations mechanism [5] and a Markov Random Field approach) for making global decisions based on local knowledge only. An overview of these are given in conjunction with an example of use in Section IV

The second stack service, shown in Figure 2, provides a means of enforcing the decision by using a self-stabilising approach to force the network to converge on the new reconfiguration. The concept of self-stabilization was originally pioneered in 1973, when Edsger W. Dijkstra [6] and the service in our platform builds on this idea [7]. Self-stabilization [8] focuses on the ability of a system to converge, within a finite number of steps, from an arbitrary state to a state that exhibits desired system behavior. The process of self-stabilization is non-terminating, it continues as long as the system, e.g. the network, persists. A self-stabilizing networking system will eventually converge to correct global behavior.

Note the stack services are services locally available and under no circumstances is Figure 2 meant to suggest any kind of centrally provided services.

\section{EXAMPLE 1: RECONFIGURING UNILATERALLY}

The potential of the reconfigurable platform presented here is illustrated with two examples. The first example focuses on reconfiguration based on a unilateral decision at the node. Components have been created for IRIS that can be used to create an Orthogonal Frequency Division Multiplexing (OFDM) transceiver and in this example two nodes communicate with each other using the OFDM transceiver. From the point of view of a node in the example, reconfiguration is triggered in two ways.

A downward push by the application determines the number of OFDM sub-carriers to be used in the communication system. (A simple rule has been set that states the minimum number of resources should be used. - currently this is treated in a simple manner with the DAWN chat application requesting 20 sub-carriers and the DAWN web application requesting 128 sub-carriers.)

Channel information creates an upward push and further specifies the sub-carriers. In this case the sub-carrier allocation scheme is designed to avoid carrier frequencies that are being subjected to strong interfering transmissions which may result in the possible unrecoverable loss of information if that frequency was used for data transmission. The communication between application and physical layer is accommodated via the blackboard as illustrated in Figure 2. A technique that enables frame synchronization, carrier-frequency offset estimation and a means of notifying the remote receiver(s) of the sub-carrier allocation using a single OFDM symbol is used. No extra signalling is needed to transmit details of the chosen transmission carriers to the receiver, resulting in an efficient system. Further details of the techniques used can be found in [9]. Should the application change or should another large interfering source appear in different frequency bands the system can react to the changes and reconfigure accordingly.

Figure 3 shows a power spectral density of the sampled signal. The rectangular box depicts the frequency band of interest in which the sub-carriers will be located. As can be seen parts of the band are experiencing high interference. The second plot in 3 indicates which part of the spectrum will not be used for sub-carriers, i.e. in terms of the OFDM process, the frequency bins in this portion will not be filled. A spectral mask is generated to inform the receiver as to what sub-carriers are used. As a result of not using sub-carriers that experience high interference the overall SNR is better and hence we can use a more efficient modulation scheme. Figure 4 gives an indication of how taking this dynamic approach is valuable.

The graph in Figure 4 depicts the results of a simple test in which two scenarios were evaluated. The first scenario is a traditional static implementation of OFDM using the 

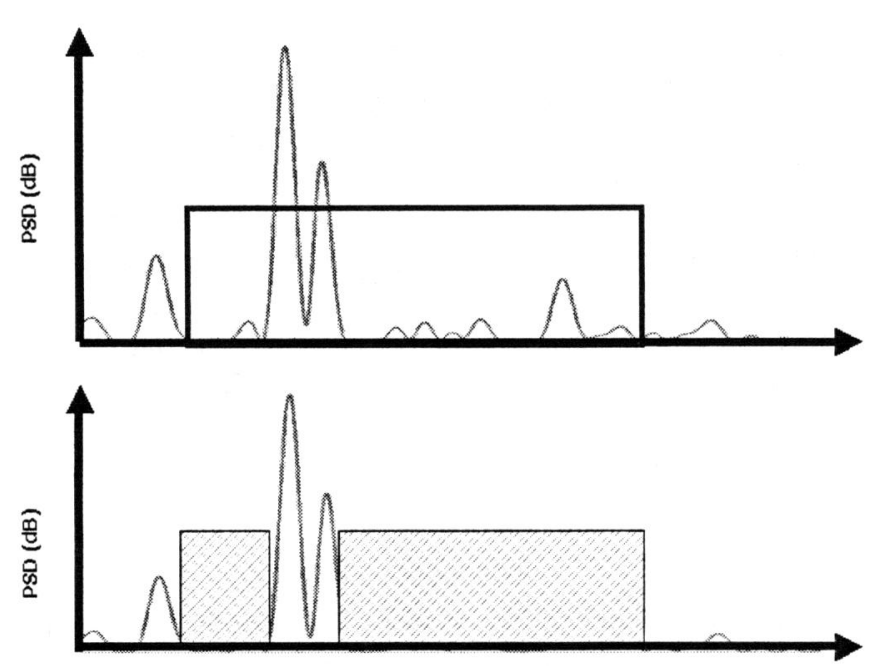

Frequency

Fig. 3. Removal of Portion of the Spectrum for Consideration

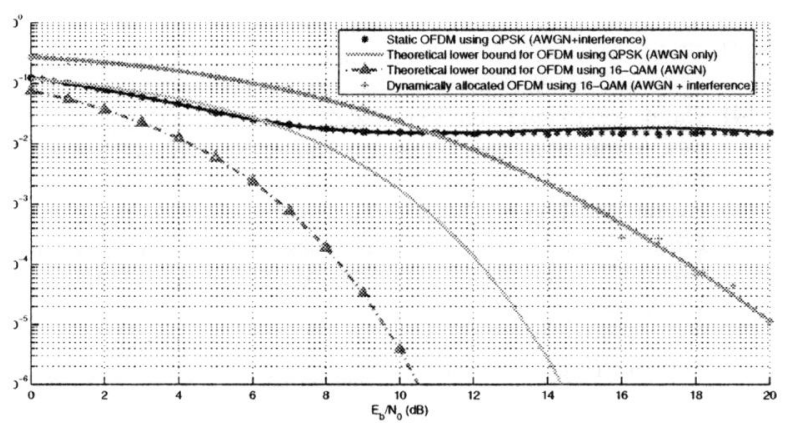

Fig. 4. BER vs $E b / N 0$ for Static and Dynamic Cases

traditional QPSK as the sub-carrier modulation technique. All of the possible 128 sub-carriers are employed regardless of the wireless channel conditions. The second scenario employs the dynamic approach using the interference avoidance sub-carrier allocation technique. Doing this means that the sub-carriers in the affected area are not used but on the sub-carriers that are used, a 16-QAM modulation technique can now be applied. In this figure a graph of the BER vs $E b / N 0$ for 1000 OFDM frames for un-coded static OFDM using QPSK on all possible sub-carriers and un-coded Dynamic OFDM using 16-QAM on valid carriers for an AWGN channel model affected by multiple-frequency FM interference sources. As can be seen the dynamic scenario offers a significant improvement.

The example here has two nodes. In the case of $n$ nodes all nodes of the network take local action only. It may be the case that by happy coincidence communication across the network is optimized. While this type of comment is obvious and on one level not very meaningful the idea of allowing a node to optimise locally is important in disaggregated systems and allows for freer development of networks.

\section{Example 2: Reconfiguring Multilaterally}

The second example focuses on an example in which a multilateral decision must be taken and involves ad hoc routing [7]. Ad hoc routing protocols are at the core of ad hoc networks allowing remote nodes to communicate on a peer-topeer basis. In our platform the Ad hoc On-demand Distance Vector routing (AODV) [10], Dynamic Source Routing (DSR) [11], and Optimised Link State Routing (OLSR) [12]) have been implemented. However from the wide body of work that exists in the area of ad hoc network routing, it has been well established in the literature that a one-size-fits-all approach with regard to the choice of optimum routing protocol does not suffice [13], [14], [15]. Rather than continuing to search for the optimal routing protocol, an alternative approach is to design a system that allows nodes to dynamically configure and utilize the most suitable ad hoc routing protocol [16] for the prevailing network conditions. In other words the actual ad hoc routing protocol in use, now becomes a variable whose value must be set across the network.

The literature has shown that there is a correlation between node mobility levels, node density levels and network traffic and the optimal routing protocol. If these parameters are known then the best protocol can be selected. In a very distributed and disaggregated network any one node has a local view only and can only get a view of the network conditions in its own vicinity. Therefore nodes in different parts of the network may have conflicting opinions as to which routing protocol is best. In order for a network of nodes with local knowledge only come to a consensus we have designed a mechanism that form part of the stack services, illustrated in Figure 2 based on a diffusion of innovation approach.

\section{A. Diffusion of Innovations}

The concept of diffusion of innovations [5] arises in the study of social and cultural behavior with regard to how innovations come to be adopted or rejected by members of a society. In the decentralized diffusion model, decisions regarding such matters as when an innovation should be diffused, how it will be diffused and how it should be evaluated are shared by the potential adopters. New ideas, or innovations, may grow out of the experience of certain individuals, or potential adopters, rather than through the specific promotion of centralized change agents. In the context of this work the innovation to be spread is the reconfiguration choice and a protocol based on the diffusion of innovations has been created to do this. The are three main elements to the protocol.

(1) Each node continually observes its networking conditions and shares that data with its neighbors. In this case the observations are about node mobility and density etc. Observations are made passively, i.e. nodes do not solicit information and there is no hand-shaking.

(2) Each node repeatedly evaluates a soft-state preference based on its observations, the observations of its neighbors and its neighbors soft-state decisions. In terms of the decision making process, the notions of Early Adopter, Early Majority, Late Majority and Laggard from the theory of diffusion of 
innovations are used. Early Adopters have a high degree of connectedness and make reasoned, evidence-based decisions. They decrease local uncertainty about an innovation by adopting it and conveying messages to other near-peers by means of interpersonal networks, i.e. neighbor to neighbor connections. The Early Majority do not lead opinion as the Early Adopter does, but through frequent interaction with their peers, they tend to follow the early adopters. The Late Majority, being sceptical, wait until most of their social system have already adopted. Laggards, as the name suggests, are the last in a social system to adopt an innovation. They have no opinion leadership qualities and are very local in outlook. Each node attempts to make an Early Adopter decision first. If it fails it tries to make one using the next model and if no model is suitable the node is a Laggard and and makes no soft-decision. The Early Majority nodes' decisions and Late majority nodes' decisions are subject to the control of individual thresholds which dictate how easily persuaded a node can be (e.g. majority of neighbors must hold same opinion for EM model).

(3) A hard-decision is made when a soft-state decision is sustained. In sum, a global decision emerges as nodes are either strong enough to make their own decision and therefore be leaders and influence others or nodes are highly influenced by others and just follow.

\section{B. Brief Analysis}

The Diffusion Of Innovations approach has been implemented fully in the reconfiguration platform and provides a mechanism for nodes to reach consensus in highly dynamic and distributed environments. Key to the success of this technique is an understanding of local observations, details of which are beyond the scope of the paper but can be found in [2]. The technique has been implemented in such a manner so as to not overburden the network therefore allowing the added complexity of reconfiguration not to be at such a high cost so as to make it undesirable.

An alternative approach is also being developed. This approach involves a Markov Random Field (MRF) Maximum $A$ Posteriori (MAP) framework to facilitate this distributed decision making process. The MRF models the interdependance of neighboring nodes and the desire to form a consensus. The MAP framework allows the nodes to incorporate local observations in the analysis. Modelling the wireless and/or mobile network as a Markov Random Field (MRF) and the subsequent use of an MRF-MAP framework provides an efficient means for making decisions in a fully distributed system in which individual nodes have only myopic views of their surroundings. Again in this situation a global decision can emerge from local observation.The MRF-MAP framework however remains at the simulation stage. Initial results show that the mechanism is a promising means of making reconfiguration decisions but the transfer of the approach to a real wireless scenario remains as a challenge.

\section{CONCLUSION}

The paper presented an overview of a platform for exploring reconfigurability is distributed and/or disaggregated networks. The platform facilitates a high degree of reconfigurability both at the physical layer and higher layers of the stack. Physical layer options include a general purpose processor software radio engine. Higher layers in the stack can be reconfigured internally or completely swapped. Mechanisms exist for reaching consensus in the case of multilateral reconfiguration choices. The purpose of the platform is to facilitate the creation of imaginative and alternative reconfigurable scenarios in order to progress the field further

\section{ACKNOWLEDGMENT}

The author would like to thank Tim Forde, Keith Nolan and Senan Doyle for their contributions. This material includes work supported by the Science Foundation of Ireland under Grant No. 03/CE3/1405.

\section{REFERENCES}

[1] Charles E. Perkins, Ad Hoc Networking, Addison-Wesley, 2001.

[2] Tim K. Forde, "Flexibility in Ad hoc Networks," in Ph.D dissertation, Trinity College Dublin, Ireland, 2005.

[3] Philip Mackenzie, "Software and reconfigurability for software radio systems," in Ph.D dissertation, Trinity College Dublin, Ireland, 2004.

[4] Hagit Attiya and Jennifer Welch, Distributed Computing: Fundamentals, Simulations and Advanced Topics, McGraw-Hill Publishing, 1998, England.

[5] Everett M. Rogers, Diffusion of Innovations, Free Press, London, 1996.

[6] Edsger W. Dijkstra, "Self-stabilizing Systems in Spite of Distributed Control", Communications of the ACM, Volume 17, No. 11, November 1973, pp643-644.

[7] T.K., Forde, L.E., Doyle, L.E. and D., OMahony, "Self-Stabilizing Network-Layer Auto-Configuration for Mobile Ad Hoc Network Nodes" in Proceedings of the IEEE International Conference on Wireless and Mobile Computing, Networking and Communications (WiMob2005),August 22nd - 24th, Montreal Canada, 2005.

[8] Shlomi Dolev, Self-stabilization,MIT Press, MA, 2000.

[9] Keith Nolan, Linda Doyle, Philip Mackenzie, and Donal O' Mahony "OFDM Sub-carrier Allocation Scheme for a Multiple User Data Enhanced Radio Server (MUDERS)," in Proceedings of the 4th Software Defined Radio (SDR) Forum Technical Conference 2004, Phoenix, Arizona, USA, 2004. vol. A, pp: 71-76.

[10] Charles E. Perkins, and Elizabeth M. Royer, "Ad hoc On Demand Distance Vector Routing", in Proceedings of the Second IEEE Workshop on Mobile Computing Systems and Applications, Philadelpia, Pennsylvania, USA, 1999, pp90-100.

[11] David B. Johnson and David A. Maltz, "Dynamic Source Routing in Ad Hoc Wireless Networks ", Mobile Computing, Kluwer Academic Publishers, 1996, pp153-181.

[12] Thomas Clausen, Philippe Jacquet, Anis Laouiti, Pascale Minet, Paul Muhlethaler, Amir Qayyum and Laurent Viennot, Optimized Link State Routing Protocol, IETF MANET Working Group, 2002.

[13] Laurent Viennot, Philippe Jacquet and Thomas Heide Clausen, "Analyzing Control Traffic Overhead versus Mobility and Data Traffic Activity in Mobile Ad-hoc Network Protocols", Wireless Networks, Kluwer Academic Press, Volume 10, No. 4, July 2004, pp447-455.

[14] Dmitri D. Perkins, Herman D. Hughes and Charles B. Owen, "Factors Affecting the Performance of Ad Hoc Networks" in Proceedings of the IEEE International Conference on Communications, Volume 4, 2002, pp2048-2052.

[15] Samir R. Das, Charles E. Perkins and Elizabeth M. Royer, "Performance Comparison of Two On-demand Routing Protocols for Ad Hoc Networks", IEEE Personal Communications, Volum 8, No. 1, February 2001, pp16-28.

[16] Jeff Boleng, William Navidi and Tracy Camp, "Metrics to Enable Adaptive Protocols for Mobile Ad Hoc Networks", in Proceedings of the International Conference on Wireless Networks, 2002, pp293-298. 